\title{
Second-year visual acuity outcomes of nAMD patients treated with aflibercept: data analysis from the UK Aflibercept Users Group
}

${ }^{1}$ Eye Unit, University Hospital Southampton NHS Foundation Trust, Southampton, UK

${ }^{2}$ Clinical and Experimental Sciences, University of Southampton,

Southampton, UK

${ }^{3}$ Gloucestershire Hospitals NHS Foundation Trust, Gloucester, UK

${ }^{4}$ Newcastle Upon Tyne Hospitals Foundation NHS Trust, Newcastle Upon Tyne, UK

Correspondence:

AJ Lotery, Division of Neurosciences, University of Southampton, Clinical and Experimental Sciences,

Faculty of Medicine, South Lab and Path Block

Mailpoint 806, Level D, Southampton SO16 6YD, UK Tel: +4402381 20 5049; Fax: +44 02381206085 .

E-mail: a.j.lotery@soton.ac.uk

Received: 26 November 2016 Accepted in revised form: 26 April 2017

Published online:

16 June 2017

Very sadly, our co-author RLJ passed away leaving behind a great heritance of significant research articles. RLJ was the Medical Director of Medisoft Limited the Electronic Medical Record Supplier to all the sites contributing to this study. This paper is dedicated to the memory of our co-worker RL

\section{Abstract}

Purpose To audit the visual acuity (VA) outcomes achieved at the end of year two in 17 UK centres, which followed the year 1 VIEW protocol in year 1, but a variable approach in year 2 for aflibercept for neovascular macular degeneration (nAMD). Patients and methods Retrospective data analysis, from an electronic medical record, of a consecutive series of treatment-naive nAMD patients who received aflibercept for 2 consecutive years, having followed the VIEW protocol in year one, defined as eyes having received 7 or 8 injections from baseline.

Results The mean number of intravitreal injections (IVI)s during year 2 was 3.7 in 1180 eyes (1083 patients). The mean baseline VA of the whole cohort was 56.3 ETDRS letters, improving to 61.3 at 1 year $(+5)$ and $59.1(+2.8)$ at the end of year 2 . The mean VA letter score at the end of year 2, stratified by number of IVIs into three groups was as follows: group A, $\mathbf{5 7 . 3}$ (gain of +1.7$)(44 \%$ of eyes $(</=3$ IVIs)); group $B$, $59.8(+3.8)(34 \%$ of eyes (4-5 IVIs)); group $C$, $61.7(+3.7)(22 \%$ of eyes $(>/=6$ IVIs $))$.

Even though there were VA gains in the three groups over the 2-years, there was a drop in VA in year one to two. Eyes that received $>/=6$ IVIs (group $C$ ) had a smaller reduction of VA during year 2 than those which received $<I=3$ IVIs (group $A$ ) $(P=0.0014)$.

Conclusions Providing a higher number of injections after a $Q 8$ regime in year 1 results in higher VA gains in year 2 of treatment. Eye (2017) 31, 1582-1588; doi:10.1038/eye.2017.108; published online 16 June 2017
H Almuhtaseb ${ }^{1,2}$, RL Johnston ${ }^{3}$, JS Talks ${ }^{4}$ and AJ Lotery ${ }^{1,2}$
Introduction

Age-related macular degeneration (AMD) is a leading cause of irreversible vision loss in the elderly in developed countries. ${ }^{1}$

While non-neovascular AMD is commoner, neovascular AMD is responsible for most cases of severe vision loss. Therapeutic antibodies directed at inhibiting Vascular Endothelial Growth Factor (VEGF) therapy are the most commonly used treatment for neovascular AMD, particularly sub-foveal neovascular lesions. $^{2}$

Aflibercept, (Eylea; Bayer Pharma AG, Berlin, Germany), is one such anti-VEGF agent. The molecule serves as a VEGF decoy to inhibit the growth of new blood vessels. Aflibercept was approved for the treatment of neovascular AMD by the FDA in 2011.

Two similarly designed, phase-3 studies (VEGF Trap-Eye: investigation of efficacy and safety in wet AMD (VIEW 1, VIEW 2)) compared monthly and every 2-month dosing of intravitreal aflibercept injections (VEGF TrapEye; Regeneron, Tarrytown, NY, USA and Bayer HealthCare, Berlin, Germany) with monthly ranibizumab. These showed that intravitreal aflibercept dosed monthly or every 2 months after 3 initial monthly doses produced similar efficacy and safety outcomes to monthly ranibizumab, with the every 2-month regimen offering the potential to reduce the risk from monthly intravitreal injections and the burden of monthly monitoring. ${ }^{3}$ Eylea's efficacy and the reduced burden on healthcare resources led to the adoption of the VIEW year 1 protocol by many NHS centres. 
Conversely, the year 2 VIEW protocol of a capped PRN regimen with monthly visits has not been widely adopted in the NHS due to the health resources needed.

In an attempt to determine the optimal real-world pathway, we audited the visual outcomes achieved at the end of year two of therapy with aflibercept in 17 UK centres to see which treatment approaches yielded the optimal visual acuity outcomes.

\section{Subjects and methods}

\section{Study design}

The anonymised data were extracted from 17 UK NHS Hospitals, as detailed in the acknowledgements section, on all treatment-naive eyes who were initiated on treatment for nAMD with aflibercept, received aflibercept according to the VIEW 1\&2 Clinical Trials' protocol during the first year of treatment and finished year 2 of treatment with aflibercept prior to the data cutoff point. All the data were recorded using a single EMR system (Medisoft Ophthalmology, Medisoft Limited, Leeds, UK), which mandated collection of a standardized dataset throughout the nAMD care pathway. The lead clinician and Caldicott Guardian (responsible nominee for the data protection) at each NHS Hospital gave written approval for the anonymised data extraction. The anonymized database analyses of this type do not require ethical permission as they are viewed as audit or service evaluation (see http:/ / www.hra.nhs.uk/researchcommunity/beforeyou-apply/determine-whether-yourstudy-is-research/). This study was conducted in accordance with the declaration of Helsinki, and the UK's Data Protection Act.

Although this study is retrospective in nature, the dataset mandated by the EMR was defined prospectively before the first data entry and hence the study methodology is somewhat closer to an electronic case report form used in clinical trials than a conventional analysis of the unstructured data in a retrospective chart review

The EMR system (Medisoft) was asked to provide the data to the United Kingdom Aflibercept Users Group to support an audit of second year visual acuity outcomes and injection frequencies in nAMD patients treated with aflibercept. The Aflibercept Users Group is a group of NHS centres that agreed to use aflibercept following the VIEW protocol for treatment-naive nAMD patients, had the same EMR (Medisoft) and were willing to pool their data.

The initial dataset included any eye with a visual acuity (actual or imputed) at 104 weeks. Results from eyes whose treatment did not follow the VIEW protocol during year 1 was excluded. Eyes following the VIEW protocol during year 1 were defined as eyes having received 7 or 8 injections in the time period up to and including week 56 (from baseline injection), consequently we avoided the data dropout from patients whose IVIs suffered from slight rescheduling during the first year of treatment (realworld scenario). Figure 1. The pathways for provision of aflibercept in year two varied between centres.

The number of first-treated eyes, second-treated eyes and eyes receiving bilateral injections was established at baseline. The mean VA values for each 4-weekly interval, up to and including 104 weeks were calculated. The mean number of injections in the second year (the second year was defined as between week 53 and week 104 inclusive) was calculated.

Eyes were stratified empirically according to the number of injections received in the second year into three different groups, which are as follows: Group A, 0-3 IVIs; Group B, 4-5 IVIs, Group C, 6 or more injections. The mean VA in the second year for each of these groups was plotted over time to establish against the VA of the patient cohorts at the following time points: week 52, week 104 .

The number and proportion of eyes that gained 5, 10 and 15 ETDRS letters were calculated in addition to the number and proportion of eyes that finished year 2 of treatment with an ETDRS letter score of $\geq 70 \mathrm{~L}$. Letter gains represent change from baseline and are cumulative that is, eyes gaining 15 letters or more are also counted in 5 and 10 letter gains.

\section{Treatment posology, dates and data variables}

Unlike in most trials, real-world patients receiving antiVEGF treatment are not always seen monthly. The VA data for each eye was divided up into monthly time

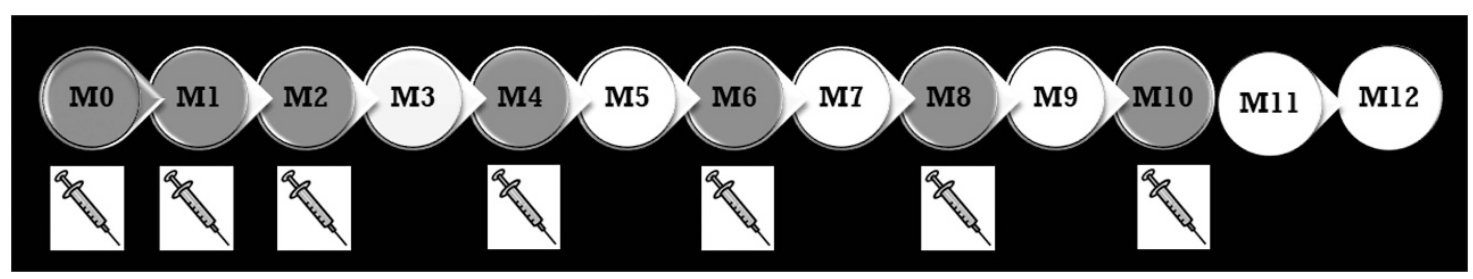

Figure 1 During year one of treatment patients followed the VIEW 1 and 2 Clinical Trials' protocol in which there were three loading IVIs (Q4W) followed by an IVI every other month. 
windows. This is how most anti-VEGF trial results have been reported. Once the data for each eye had been divided up into monthly time windows, each 'empty' monthly time window was populated with the average (mean) VA of the months either side of it. Where an eye had more than one empty month in succession, all of these empty months would be populated with the average (mean) VA of the months on either side of this time period. No data imputation was applied beyond the time of last known follow-up. We used the same principle in the previously published work of the National UK Aflibercept Group, which discussed the year 1 data. 4 Unlike clinical trials, no observations were carried forward beyond the last recorded. The data cutoff point was 25 July 2016.

At baseline, an optical coherence tomography scan (SDOCT) was carried out. To further confirm the diagnosis, fundus fluorescein angiography and indocyanine green angiography were carried out in the majority of eyes.

At each visit (including baseline), best-corrected visual acuity (BCVA) using each patient's habitual correction, injection procedure and the follow-up data were entered into the EMR system used (Medisoft). This is routine clinical practice in the centres that form this cohort of Aflibercept Users Group. In many centres a paperless system was implemented, in others a hybrid paperless and paper notes system was used. Ocular and systemic pre-, intra- and post-operative complications were entered after each procedure.

\section{Visual acuity and missing value imputation}

At each visit, the best-measured visual acuity value was used in the analysis. Most visual acuity values were recorded using the patients' habitual correction rather than with refraction. Values corresponding to count fingers $(\mathrm{CF})$, hand movements (HM), perception of light (PL) and no PL (NPL) were substituted with values of 0 ETDRS letters. ETDRS (early-treatment diabetic retinopathy study) charts were used to record visual acuity letter scores at $2 \mathrm{~m}$. In cases where an ETDRS letter score chart was not used, visual acuity (Snellen or LogMAR) was converted into an ETDRS letter score for analysis.

\section{Primary and secondary outcomes}

The primary outcome measures were mean change in ETDRS BCVA at year 1 and at the end of year 2 .

Secondary outcomes included the mean number of IVIs patients received during year 2 of treatment. Eyes were stratified according to the number of injections received in the second year into three groups as follows: Group A, 0-3 IVIs; Group B, 4-5 IVIs, Group C, 6 or more injections.
T-test and ANOVA for independent samples were used to compare means of BCVA and number of IVIs between different groups. $P$-value of $\leq 0.05$ was considered to be statistically significant.

\section{Results}

\section{Participants}

The data were extracted from the electronic patient records of 17 NHS centres. A total of 1180 eyes of 1083 patients were retrospectively recruited. In all, 864 eyes were first-treated eyes, 180 were second-treated eyes and 136 eyes received bilateral injections at baseline. Eyes were not classified as first or second treated if both eyes were initiated on treatment on the same day or if the time difference between the baseline IVIs in both eyes was less than or equal to a 2 -week interval. The mean age at baseline injection was 82 years. $68 \%$ of patients were female.

\section{Visual acuity, injection frequency}

The mean baseline visual acuity (VA) at month 0 of the whole cohort was 56.3L improving to 59.1L (+2.8L gain) at end of year 2. The mean change in VA at week 52 (61.3L) compared to baseline (56.3L) \{mean gain in year 1 \} was $+5 \mathrm{~L}$ of the whole cohort. The mean change in VA at week 104 (59.1L) compared to week 52 (61.3L) (mean change in year 2) was -2.2L. Figure 2.

Any eye that did not follow the VIEW protocol during year 1 was excluded. Eyes following the VIEW protocol during year 1 were defined as eyes having received 7 or 8 injections in the time period up to and including week 56 (from baseline injection). A variable approach (multiple dosing strategies) was implemented during year 2 of treatment with aflibercept. Variable treatment regimens were implemented in different centres in year 2: fixed

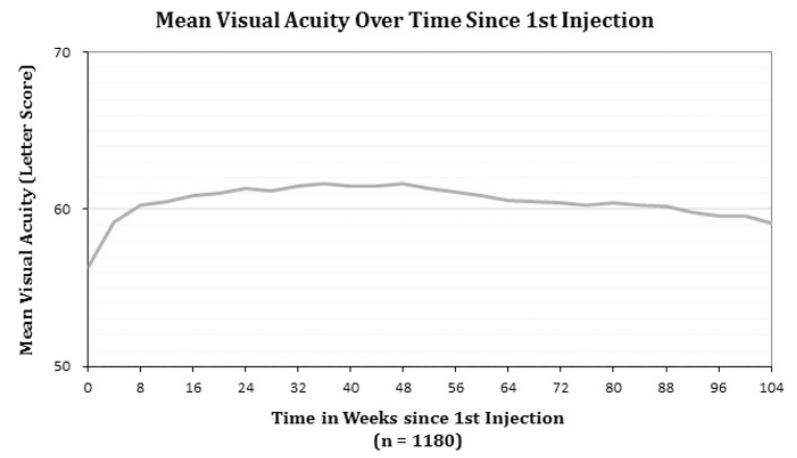

Figure 2 Mean VA (ETDRS) over time since the first IVI. A mean gain of $+2.8 \mathrm{~L}$ was observed during the 2-year treatment period. A mean gain of $+5 \mathrm{~L}$ was observed in year 1 . A mean loss of $-2.2 \mathrm{~L}$ was observed in year 2, when VA at week 104 was compared to VA at end of year 1. 
dosing on a schedule and variable dosing guided by visual acuity criteria, dilated fundus exam findings, optical coherence tomography (OCT) and fundus fluorescein angiographic (FFA) changes, suggesting activity were implemented.

During year 2, the mean number of injections in all 1180 eyes was 3.7. The vast majority of this cohort of patients received a number of IVIs that ranged from 3 to 6 . Figure 3 shows the distribution of the number of IVIs patients received during year 2 of treatment.

\section{Stratification of patients based on the number of injections received in year 2}

Patients were stratified by number of IVIs received during year 2 into 3 groups as follows: group A, $44 \%$ of eyes $(n=515)$ received $(</=3$ IVIs); group $\mathrm{B}, 34 \%$ of eyes $(n=400)$ received (4-5 IVIs); group C, $22 \%$ of eyes $(n=265)$ received $(>/=6$ IVIs). The mean baseline VA of groups A, B and C were 55.6, 56.0 and 58.0L, respectively. Group C, which received the higher number of IVIs during year 2 had a slightly higher baseline VA.

The mean VA at the end of year 1 of groups $\mathrm{A}, \mathrm{B}$ and $\mathrm{C}$ were $60.1 \mathrm{~L}, 62.2 \mathrm{~L}$ and $62.4 \mathrm{~L}$, respectively, giving a mean gain of $+4.5 \mathrm{~L},+6.2 \mathrm{~L}$ and $+4.4 \mathrm{~L}$.

The mean VA at the end of year 2 of groups A, B and C were 57.3L, 59.8L and 61.7L, respectively. A mean gain of $+1.7 \mathrm{~L},+3.8 \mathrm{~L}$ and +3.7L was seen from baseline. (Table 1).

Even though there was a visual acuity gain in the three groups at the end of the 2-year treatment period, compared to baseline, eyes suffered a drop of VA from the 1 year point. A mean loss of $-2.8 \mathrm{~L},-2.4 \mathrm{~L}$ and $-0.7 \mathrm{~L}$ in groups A, B and C, respectively. Eyes that received $>$ / $=6$ IVIs (group C) suffered less reduction in visual acuity during year 2 . When this reduction in visual acuity (group C) was compared to that observed in eyes, which received $</=3$ IVIs (group A), the difference was statistically significant $(P=0.0014$, ANOVA analysis).

In our cohort, the $(n)$ and proportion of eyes which gained $>/=5,>/=10$ and $>/=15$ letters from baseline to week 104 were (571) 48\%, (387) 33\% and (247) $21 \%$. The $(n)$ and proportion of eyes which finished year 2 at week 104 with a BCVA of $\geq 70$ ETDRS L were (401) $34 \%$.

In our cohort, the $(n)$ and proportion of eyes, which lost $>/=5,>/=10$ and $>/=15$ letters from baseline to week 104 were (311) 26\%, (208) 18\% and (145) 12\%. Letter gains and losses represent change from baseline and are cumulative that is, eyes gaining 15 letters or more are also counted in 5 and 10 letter gains; Eyes achieving 70 letters and above are at time point. The same applies to ETDRS letter losses.

Figure 4 shows VA over time of the whole cohort stratified by the number of 2 nd year IVIs given.

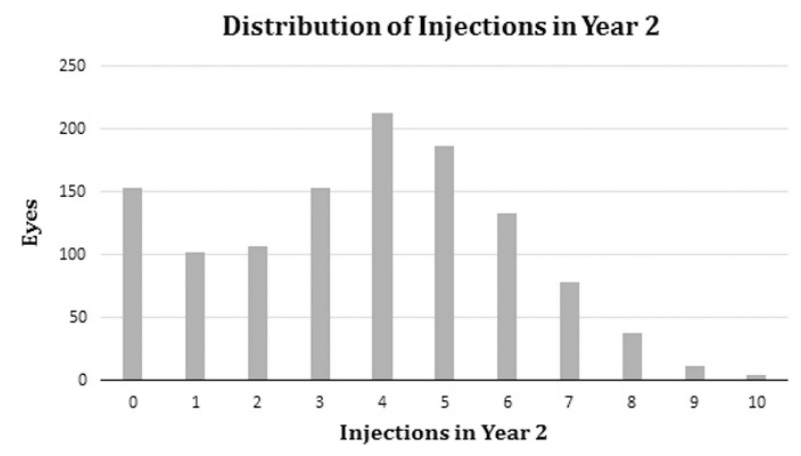

Figure 3 The distribution of IVIs in year 2, patients having received 7-8 IVIs in year 1, following the VIEW protocol. In year 2 , treatment regimens varied per centre.

\section{Discussion}

The results from the follow-up regimen of mandatory quarterly dosing with intervening as-needed injections (capped PRN) in the second year of the VIEW studies confirm the sustained improvements in visual acuity, central retinal thickness and CNV size achieved by fixeddosing regimens of intravitreal aflibercept during the first year. $^{3}$

In VIEW studies, during the follow-up period from weeks 52 to 96 (year 2), patients continued to receive the same dose of study drugs as in the first 52 weeks, but received injections at least every 12 weeks, with monthly evaluations for interim injections based on prespecified retreatment criteria (mandatory quarterly dosing with examination guided interim injections or capped-PRN).

In our cohort of patients, the mean letter gain in year 1 was $+5 \mathrm{~L}$, comparable to the $+5.1 \mathrm{~L}$ gain observed in a previous report carried out by the same national UK Aflibercept Users Group previously published in Ophthalmology. ${ }^{4}$

This compares to $+8.4 \mathrm{~L}$ in the integrated analysis of the VIEW 1 and VIEW 2 studies, it being 7.9L in VIEW 1 and 8.9L in VIEW 2. In the VIEW study, the baseline vision was $53.6 \mathrm{~L}$ and the mean one-year visual acuity was 62L, giving a gain of +8.4 letters. The mean baseline visual acuity in our cohort of patients was 56.3L and at week 52 $61.3 \mathrm{~L}$, so a similar actual mean visual acuity, albeit it with a smaller rise of $+5 \mathrm{~L}$.

These results are encouraging as replicating outcomes of a clinical trial in a real-world scenario is problematic. In clinical practice, the patient group is likely to be more heterogeneous compared to a clinical trial. Differences include larger CNV lesions $>12$ disc areas, $>50 \%$ of $\mathrm{CNV}$ lesions being haemorrhagic or having atrophic or fibrotic components at baseline, broader baseline VA measurements, delays in treatment, more ocular comorbidities such as epi-retinal membranes or cataract and the patients having a greater range of co-morbidities. 
Table 1 Different measurements of the IVI-stratified 3 groups

\begin{tabular}{llll}
\hline & A: $<=3$ injections in year 2 & B: 4-5 injections in year 2 & C: $>=6$ injections in year 2 \\
\hline Count (no. of eyes) & 515 & 400 & 265 \\
Mean VA at baseline (SD) & $55.6 \mathrm{~L}(14.2)$ & $56.0 \mathrm{~L}(13.7)$ & $58.0 \mathrm{~L}(13.4)$ \\
Mean VA at W52 (SD) & $60.1 \mathrm{~L}(16.5)$ & $62.2 \mathrm{~L}(14.5)$ & $62.4 \mathrm{~L}(13.5)$ \\
Mean VA at W104 (SD) & $57.3 \mathrm{~L}(19.4)$ & $59.8 \mathrm{~L}(16.4)$ & $61.7 \mathrm{~L}(15.1)$ \\
$\Delta$ VA end of year 1 (to baseline) & $+4.5 \mathrm{~L}$ & $+6.2 \mathrm{~L}$ & $+4.4 \mathrm{~L}$ \\
$\Delta$ VA end of year 2 (to baseline) & $+1.7 \mathrm{~L}$ & $+3.8 \mathrm{~L}$ & $+3.7 \mathrm{~L}$ \\
$\Delta$ (W104-W52) & $-2.8 \mathrm{~L}$ & $-2.4 \mathrm{~L}$ & -0.7 \\
$\Delta$ (W104-M52) & $\mathrm{A}$ vs B & $P=0.0014$ & $\mathrm{~B}$ vs C \\
& $P=0.0399$ & $P=0.1359$ \\
\hline
\end{tabular}

Mean Visual Acuity Over Time Stratified by Number of Injections in Year 2

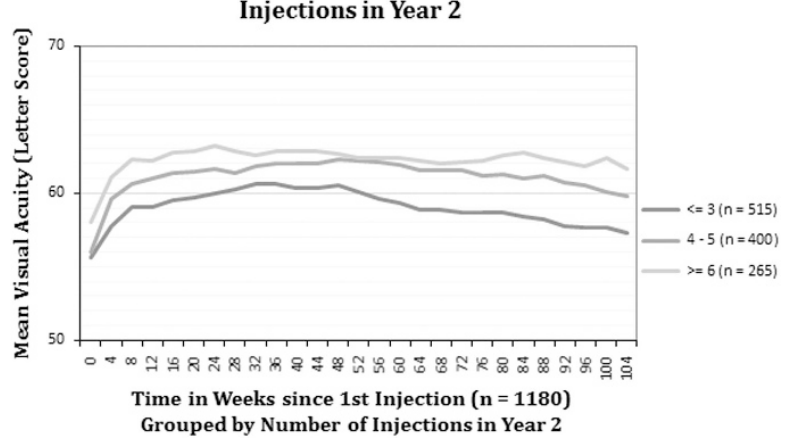

Figure 4 Mean VA over time stratified by \# of IVIs in year 2.

At the end of year two, the mean gain of VA had reduced to $+2.8 \mathrm{~L}$ so a mean loss of $-2.2 \mathrm{~L}$.

In VIEW studies, at week 96, the mean BCVA gain was 7.6 letters in the $2 \mathrm{q} 8$ group; ${ }^{5}$ this gain represented approximately a 1 letter loss during the capped PRN (modified quarterly dosing) phase. Overall, 30.9\% of patients in 2q8 group gained 15 letters or more from baseline to week 52 in the VIEW studies. ${ }^{5}$ The proportion of patients who gained 15 letters or more from baseline to week 96 was 33.4\% in the aflibercept Q8W group.

In our cohort, the $(n)$ and proportion of eyes, which gained $\geq 15$ letters from baseline to week 104 were (247) $21 \%$. The $(n)$ and proportion of eyes, which finished year 2 at week 104 with a BCVA of $\geq 70$ ETDRS L were (401) $34 \%$.

Eyes that received $>/=6$ IVIs (group C) suffered less reduction in visual acuity during year 2 . When this reduction in visual acuity (group C) was compared to that observed in eyes which received $</=3$ IVIs (group A), the difference was statistically significant $(P=0.0014)$ Figure 4.

In our cohort, we recruited retrospectively only those patients who followed the VIEW protocol during year 1. Debate continues regarding what care pathway is appropriate in year 2, and whether to continue a fixeddosing, treat and extend or a PRN regimen. Some use treat and extend from the beginning of year $1 .^{6}$
Richard et al. ${ }^{7}$ in multiple post hoc analyses of VIEW trial patients, showed that after W52, $\sim 20 \%$ of patients lost five Early Treatment Diabetic Retinopathy Study (ETDRS) letters or more across all treatment arms with PRN treatment. Patients who met the retreatment criterion of loss of five ETDRS letters or more in the first quarter of the PRN dosing phase did not recover; mean final VA loss across the four study arms was -4.4 to -5.8 letters.

A limitation of this study is that it is not known why different injection frequencies were given as patients were treated in 17 centres following individualised protocols beyond year 1 . One explanation could be that those patients given less injections were dry and had more atrophy, others could be that the centre had capacity issues or the patients were unable to attend all the scheduled visits or did not wish to have multiple injections. In other studies, ${ }^{8-10}$ with a continuous treatment arm, VA was maintained at the end of year 2.

In the ANCHOR study (0.5 mg Ranibizumab arm), a $+11.3 \mathrm{~L}$ gain was observed at week 52 compared to baseline. At week 104, there was a $+10.7 \mathrm{~L}$ gain compared to baseline; an average loss of $-0.6 \mathrm{~L}$ was observed in the arm that received ranibizumab monthly for two consecutive years. ${ }^{8}$

In the CATT study, at week 104, the mean increase in letters of visual acuity from baseline was $+8.8 \mathrm{~L}$ in the ranibizumab-monthly group, ${ }^{9}$ the mean increase at week 52 compared to baseline was $+8.5 \mathrm{~L}$; the mean visual acuity among patients assigned to continue monthly treatment changed little during Year $2(-0.3 \mathrm{~L})$, while the mean change in the groups switched from monthly to treatment as needed were -1.8 letters in ranibizumabtreated patients. ${ }^{10}$

Switching to as-needed dosing after 1 year of monthly treatment, with either drug (ranibizumab/bevacizumab) in the CATT trial, produced a mean 2.2 letter decrease, yielding mean visual acuity nearly equal to that obtained with as-needed dosing for 2 years. 
This may be due to more lesion growth, fluorescein leakage, and residual fluid on OCT in eyes in the asneeded group.

Anti-VEGF therapy has significantly improved the quality of life of patients on treatment for nAMD. The biggest challenge is providing the treatment effectively for a population of patients within the constraints of finite capacity of any healthcare system.

In this study, a regimen involving a higher number of IVIs resulted in a better VA score at the end of year 2. In addition to that, these results demonstrate the need for aggressive treatment in year 2 to maintain the treatment gains achieved in year 1 of therapy with aflibercept.

We postulate that if patients had continued the fixeddosing Q8W in the second year of treatment they would have been more likely to maintain the visual acuity gains observed during year 1 . Such a regimen could lead to better outcomes with the minimum number of monitoring visits. In our cohort, an average number of IVIs in year 2 was 3.7, with the majority of patients having received (3-6 IVIs). In all, $27 \%$ of patients in our cohort received 5-6 IVIs in year 2. Implementing a fixed, Q8W proactive dosing regimen with aflibercept (requiring 6 injections) in year 2 would approximate the (5-6 IVIs) given reactively but with reduced monitoring. A fixed-dosing $\mathrm{Q} 8 \mathrm{~W}$ regimen is a proactive regimen that could be considered in a good proportion of patients in year 2 of treatment, but may still under treat some patients and might risk over treating others. A proactive pathway should be considered for patients who have finished year 1 of the VIEW pathway.

\section{Summary}

What was known before

- In this manuscript, our continuously growing Research Group to which new centres have affiliated this year -our Group- continues its work and audits the visual acuity (VA) outcomes achieved at the end of year 2 of nAMD treatment with Aflibercept (Eylea) following the year 1 VIEW protocol in year 1 but a variable approach in year 2 , addressing the issue of the frequency of injections in year 2 and its implications for the maintenance of the visual and anatomic gains achieved in year 1 .

What this study adds

- In this study, a regimen involving a higher number of IVIs in year 2 resulted in a better VA score at the end of year 2 .

- In addition to that, these results demonstrate the need for aggressive treatment in year 2 to maintain the treatment gains achieved in year 1 of therapy with Aflibercept.

\section{Conflict of interest}

Medisoft and Newcastle Upon Tyne Hospitals NHS Foundation Trust received support for the data extraction and analysis from Bayer. Bayer were not involved in the data collection, analysis or writing of this paper. The authors have received travel bursaries and attended advisory boards for Bayer, Novartis and Allergan. RLJ was the Medical Director of Medisoft Limited, the Electronic Medical Record Supplier to all the sites contributing to this study.

\section{Aflibercept users group}

A Kamal, Aintree University Hospitals NHS Foundation Trust; B Paul Barking, Havering and Redbridge University Hospitals NHS Trust; A Lobo, Bedford Hospital NHS Trust; F Ghanchi, Bradford Teaching Hospitals NHS Foundation Trust; S Mahmood, Central Manchester University Hospitals NHS Foundation Trust; N Patel, East Kent Hospitals University NHS Foundation Trust; Q Kashif, East Sussex Hospitals NHS Trust; C Santiago, Grampian NHS; G Walters, Harrogate and District NHS Foundation Trust; S Sivaprasad, King's College Hospital NHS Foundation Trust; M McKibbin, Leeds Teaching Hospitals NHS Trust; M Tahir, Royal Berkshire NHS Foundation Trust; B Musta, Sandwell and West Birmingham Hospitals NHS Trust; J Talks, The Newcastle Upon Tyne Hospitals NHS Foundation Trust; A Lotery, University Hospital Southampton NHS Foundation Trust; C Bailey, University Hospitals Bristol NHS Foundation Trust; U Chakravarthy, Queen's University Belfast

\section{References}

1 Bourne RR, Jonas JB, Flaxman SR, Keeffe J, Leasher J, Naidoo $\mathrm{K}$ et al. Prevalence and causes of vision loss in highincome countries and in Eastern and Central Europe: 1990- 2010. Br J Ophthalmol 2014; 98(5): 629-638.

2 Sarwar S, Clearfield E, Soliman MK, Sadiq MA, Baldwin AJ, Hanout $\mathrm{M}$ et al. Aflibercept for neovascular age-related macular degeneration. Cochrane Database Syst Rev 2016; 2: CD011346.

3 Heier JS, Brown DM, Chong V, Korobelnik JF, Kaiser PK, Nguyen QD et al. Intravitreal aflibercept (VEGF Trap-Eye) in wet age-related macular degeneration. Ophthalmology 2012; 119: 2537-2548.

4 Talks JS, Lotery AJ, Ghanchi F, Sivaprasad S, Johnston RL, Patel $\mathrm{N}$ et al. First-year visual acuity outcomes of providing aflibercept according to the VIEW study protocol for agerelated macular degeneration. Ophthalmol 2016; 123(2): 337-343.

5 Schmidt-Erfurth U, Kaiser PK, Korobelnik J-F, Brown DM, Chong V, Nguyen QD et al. Intravitreal aflibercept injection for neovascular age-related macular degeneration: ninetysix-week results of the VIEW studies. Ophthalmology 2014; 121(1): 193-201.

6 Barthelmes D, Campain A, Nguyen P, Arnold JJ, McAllister IL, Simpson JM et al. Effects of switching from ranibizumab to aflibercept in eyes with exudative age-related macular degeneration. Br J Ophthalmol 2016; 100(12): 1640-1645. 
7 Richard G, Monés J, Wolf S, Korobelnik JF, Guymer R, Goldstein $\mathrm{M}$ et al. Scheduled versus Pro Re Nata Dosing in the VIEW Trials. Ophthalmology 2015; 122(12): 2497-2503.

8 Brown DM, Michels M, Kaiser PK, Heier JS, Sy JP, Ianchulev T. Ranibizumab versus verteporfin photodynamic therapy for neovascular age-related macular degeneration: Two-year results of the ANCHOR study. Ophthalmology 2009; 116: 57-65.e5.
9 Comparison of Age-related Macular Degeneration Treatments Trials (CATT) Research Group, Martin DF, Maguire MG, Fine SL, Ying GS, Jaffe GJ et al. Ranibizumab and bevacizumab for treatment of neovascular age-related macular degeneration: Two-year results. Ophthalmology 2012; 119: 1388-1398.

10 CATT Research Group. Ranibizumab and bevacizumab for neovascular age-related macular degeneration. $N$ Engl J Med 2011; 364: 1897-1908. 